\title{
LOS SUELOS DE LA REGION DEL AMAZONAS SE GUN UNIDADES FISIOGRAFICAS
}

Rodríguez A chung Fernando ( $\left.{ }^{*}\right)$

Bendayan A costa L eón $\left({ }^{* *}\right)$

Rojas Rodríguez Carlos $\left({ }^{* * *}\right)$

Calle Barco Carlos $\left(^{* * * *}\right)$

\section{INTRODUCCION}

Los diversos eventos geológicos sucedidos en la Región del A mazonas y la subsecuente acción de los factores climáticos e hidrológicos, han originado relieves típicos con rasgos diferenciales para cada forma de tierra. La interpretación de estas geoformas, con el propósito de diferenciar unidades edáficas, se realiza mediante el análisis fisiográfico, que consiste básicamente en el estudio detenido del modo o patrón de drenaje, grado de disectación de la superficie de la tierra, sedimentación, relieve topográfico, vegetación, litología y otros elementos fotoidentificables que permitan reconocer las diferentes formas de tierra a través de la técnica de fotointerpretación de pares estereoscópicos, permitiendo así, obtener una visión clara y sistemática de las diferentes unidades fisiográficas. Estas unidades sirven de base para la ejecución de los estudios de suelos.

El propósito de este trabajo, es identificar y sistematizar las diferentes unidades fisiográficas comprendidas en los diversos estudios de inventario y evaluación de suelos realizados en la Región del Amazonas, y correlacionarlos con las unidades edáficas existentes en este espacio geográfico.

La distribución de los suelos en las diferentes unidades fisiográficas facilita la comprensión de la problemática de este recurso, por parte de los investigadores, extensionistas, estudiantes y usuarios en general.

\section{FISIOGRAFIA DE LA REGION DEL AMAZONAS}

En la Región del A mazonas se han identificado tres (3) grandes paisajes que dominan el escenario fisiográfico: A luvial, Colinoso y M ontañoso. 


\subsection{Paisaje aluvial:}

Este paisaje, caracterizado principalmente por su topografía casi plana, está conformado por sedimentos aluviónicos del Cuaternario, tanto recientes como antiguos, los mismos que han sido acarreados y depositados dominantemente por los tributarios del río A mazonas, que tiene origen andino y, en menor proporción, por los ríos que nacen en el mismo llano amazónico.

En este paisaje se ubican las formaciones vegetales denominadas aguajales (M auritia flexuosa), principalmente en áreas depresionadas, de mal drenaje de los diferentes niveles de terrazas.

\subsubsection{Sub paisaje de llanura inundable:}

Está conformado por sedimentos aluviónicos recientes, que han sido depositados por los cauces fluviales primarios y secundarios. A barcan todas las tierras planas con pendientes menores de $2 \%$ que sufren inundaciones periódicas anuales mayormente, a excepción de las áreas que albergan palmeras hidrofíticas y que, durante la mayor parte del año, se encuentran inundadas, así como las lagunas y la mayoría de meandros, que presentan espejos de agua en forma permanente.

\section{Las unidades fisiográficas identificadas en este subpaisaje son las siguientes:}

- Islas. - Estas unidades están conformadas por tierras rodeadas de agua, siendo constituidas en su mayoría por sedimentos que varían en granulometría y que soportan inundaciones moderadas a severas.

- Orillares.- Estas unidades se encuentran localizadas principalmente a orillas de los ríos; están surcadas por líneas suavemente curvadas que ofrecen el aspecto de barras semilunares, originadas por migraciones temporales de los sedimentos acarreados por las aguas de los ríos en épocas de creciente y que, por pérdida de la velocidad de flujo, dejan sedimentaciones progresivas en forma de camellones, muy suavemente curvados, que alternan muchas veces con cursos temporarios o abandonados de ríos y quebradas, conocidos vulgarmente como cochas.

- Terrazas bajas de inundación periódica. E stán constituidas por sedimentos fluviónicos de naturaleza "arcillosa", Ias que por su escasa altura con respecto al nivel del río (menos de $2 \mathrm{~m}$.), están expuestas a soportar impactos de inundaciones, cuyas intensidades varían de moderadas a fuertes y ocasionadas por las crecientes normales de los ríos en épocas de mayores precipitaciones pluviales. 
-Terrazas bajas de inundación eventual.- Son tierras planas que se desarrollan mayormente en áreas aluviónicas, encontrándose conformadas por deposiciones de materiales 'arcillo-limosos", fundamentalmente. En cuanto a su nivel con respecto al río, se encuentran en niveles ligeramente superiores que las unidades anteriores, condición que les permite que solo sean inundadas por crecientes grandes.

\subsubsection{Sub paisaje de llanura no inundable:}

Está constituido por sedimentos aluviónicos antiguos y comprende las te razas que han alcanzado una altura que no permite que sean inundadas por las aguas de los ríos.

L as unidades fisiográficas reconocidas en este subpaisaje son:

- Terrazas medias. - Esta unidad fisiográficamente se distribuye en la zona d e influencia de los principales ríos, se caracterizan por encontrarse libres de inundaciones fluviales, pudiendo presentar relieves ligeramente de presionados que favorecen la acumulación del agua de lluvia; asimismo, presentan relieves planos a ligeramente inclinados. Se encuentran conformados por materiales moderadamente finos a finos, de origen aluvial antiguo.

- Terrazas altas. - Constituyen los pisos mas altos de las terrazas, fluctuando su altura sobre el nivel de los ríos entre 30 - $50 \mathrm{~m}$. Se derivan de materiales antiguos, alcanzando un mayor nivel de desarrollo generalmente, están formados por elementos finos, presentando un relieve uniforme, con pendientes promedio de 2 a $8 \%$. Diversos grados de disección se observan en esta unidad.

\subsection{Paisaje colinoso:}

Este paisaje se caracteriza por presentar un relieve ondulado, constituido principalmente por materiales arcillosos de escasa consolidación. Presenta gradientes suaves pero constantes y sus pendientes son del 15 al $70 \%$. Los procesos que han actuado en este paisaje están ligados generalmente a procesos de naturaleza tectónica y a procesos exógenos activos, constituidos principalmente por erosión hídrica que, en épocas pasadas, han actuado intensamente, pero que en la actualidad, su dinámica ha disminuido sustancialmente, debido a la existencia de una cobertura vegetal de naturaleza arbórea. 


\subsubsection{Sub paisaje de colinas bajas:}

Contiene formas de tierras cuyas alturas no sobrepasan los $40 \mathrm{~m}$., de acuerdo a su grado de disectación y por efecto de la erosión hídrica.

Las unidades fisiográficas identificadas en este subpaisaje son las siguientes:

- Lomadas.- Son geoformas de configuración algo redondeadas y de baja altitud, cuyas alturas fluctúan entre 5 y $20 \mathrm{~m}$. En relación a su nivel de base local. El relieve topográfico es suave; están constituidas por arcillas inconsolidadas, con gradientes que varían de 8- $15 \%$, siendo un tanto más pronunciadas en aquellas zonas transicionales a colinas. Presentan cimas redondeadas y pendientes dominantemente cortas.

- Vallecitos intercolinosos. - Se desarrollan en ambientes de colinas bajas y lomas, debido a la intensidad de la disectación, motivada por la acción de las Iluvias, las cuales van enrodando gradualmente las laderas de las colinas, propiciando así un ligero ensanchamiento en la base, debido a la deposición del material rodado y a la incapacidad de transportarlo, formando en consecuencia vallecitos de fondo plano y en forma de $\mathrm{V}$.

- Colinas bajas.- Estas unidades fisiográficas presentan cimas redondeadas a ligeramente angulosas, con alturas de $20-40 \mathrm{~m}$. y pendientes variables entre 15 y $25 \%$. Están constituidas por material arcilloso del Terciario.

\subsubsection{Sub paisaje de colinas medias:}

Presentan cimas bastante angul osas y laderas largas, con alturas de $40-70 \mathrm{~m}$. y pendientes que oscilan entre 25-50\%. Están constituidas por material arcilloso consolidado, del Terciario continental, presentando un mayor grado de erosión. Las unidades fisiográficas se diferencian por su grado de disectación.

\subsubsection{Sub paisaje de colinas altas:}

Están constituidas por dos (2) unidades fisiográficas, clasificadas únicamente por el grado de disectación (moderado y fuerte), cuyas alturas varían entre 70 y $100 \mathrm{~m}$. y sus pendientes son del orden de $50 \mathrm{a}$ $70 \%$. 


\subsection{Paisaje montañoso:}

Está formado por los cerros bajos, ubicados al sur y sureste, en la frontera con Brasil y en el área de Contamana, y por las cadenas montañosas de la vertiente oriental de los Andes, localizadas al sur y noroeste del territorio de la Región del A mazonas. Presentan gran relieve y notable escarpamiento, cuyas alturas superan los $150 \mathrm{~m}$. para los cerros y $500 \mathrm{~m}$. para las cadenas montañosas; sus pendientes son mayores del $70 \%$.

\section{LOS SUELOS DE LA REGION DEL AMAZONAS}

En todo estudio de suelos, con fines de clasificación y delimitación cartográfica, están implícitos dos conceptos fundamentales: el primero, donde prima el concepto científico y taxonómico, se refiere al estudio de los suelos en si mismo, como formaciones o cuerpos naturales diferenciables por su génesis o evolución, morfología y características: clima, vegetación, material parental, topografía y tiempo. En cambio, en el segundo prevalece el concepto técnico o práctico, se refiere al estudio de los suelos como medio apropiado para el crecimiento y desarrollo económico de las plantas de cultivo.

\subsection{Clasificación taxonómica}

De los diez (10) órdenes que comprende el Soil Taxonomy (USDA), en la Región del A mazonas se han identificado siete órdenes, cuyas características se mencionan a continuación.

- Entisoles. - Son suelos de formación reciente, que no reflejan la influencia de los factores pedogenéticos 0 solo presentan un comienzo de horizontes que están debilmente expresados, y que no cumplen los requisitos de ninguno de los horizontes de diagnóstico; presentan una morfología estratificada, sobre todo en depósitos aluviales o suelos derivados de ellos. La fertilidad de estos suelos es variable, dependiendo del material original acarreado por los ríos, siendo de mayor fertilidad natural aquellos suelos de materiales de origen andino.

En la Región se han identificado tres (3) subgrupos: Tropofluvent típico, Tropofluvent ácuico y Tropofluvent lítico.

- Inceptisoles.- Son los suelos con uno o mas horizontes de diagnóstico, que se supone se formaron más bien rápidamente y que no presentan iluviación 0 eluviación o una erosión extrema. En la mayor parte se encuentran en áreas jóvenes, pero no recientes, de ahí el nombre de Inceptisol, que es derivado del latín Inceptum, que quiere decir 'comienzo'. 
No se puede dar ninguna descripción de los ambientes que sean verdaderamente representativos de todos los Inceptisoles, pero sí se puede dar las características que son representativas: $M$ aterial original muy reciente, posiciones extremas en el paisaje, o sea tierras de pendientes y depresiones, y superficies geomórficas tan jóvenes que

limitan el desarrollo de los suelos, presentando rasgos que indican una falta de madurez edafológica.

En la Región se han identificado seis (6) sub grupos: Distropept típico, Tropacuept aérico, Tropacuept típico, Tropacuept hístico, E utropept lítico y Eutropept típico. Siendo el Eutropept (típico y lítico) los subgrupos que presentan una mayor fertilidad natural. En muchas de las áreas donde predominan Tropacuept hístico o Tropacuept típico, se reporta la presencia de la palmera aguaje (M auritia flexuosa).

- Alfisoles.- Los Alfisoles son suelos que se asemejan a los Ultisoles, pero tienen una menor acidez y son de fertilidad natural superior (con más de 35\% de saturación de bases).

\section{Tropudalf.}

En la Región se han identificado dos (2) grandes grupos: Paleudalí y

- Ultisoles.- Los Ultisoles son suelos rojos y amarillos que presentan un horizonte argílico (20\% de aumento en el contenido de arcilla en la sección de control), y que presentan una baja fertilidad natural con menos del $35 \%$ de saturación de bases, lo que le diferencia de los Alfisoles. Los Ultisoles están comprendidos principalmente dentro del rango de climas húmedos, pero abarcando desde las zonas templadas hasta los trópicos; las tierras donde se encuentran por lo común son antiguas.

En la Región del A mazonas se han identificado dos (2) sub grupos: Tropudult típico y Paleudult típico.

- Histosoles.- Los Histosoles son suelos orgánicos que se forman siempre que la producción de materia orgánica sobrepasa su mineralización, por lo común en condiciones de saturación casi continua con agua, que impide la circulación del oxígeno del suelo. Generalmente se encuentran en terrazas bajas de inundación periódica, cubiertas por palmeras donde predomina el aguaje (M auritia flexuosa).

En la Región se ha identificado un subgrupo: Tropofibrist hídrico.

- Espodosoles.- Los Espodosoles o Podzoles son suelos sumamente arenosos y ácidos, con tan baja fertilidad natural que no son capaces de soportar un bosque húmedo tropical o en su defecto soportan bosques de poca utilidad. 
En la Región se ha identificado un subgrupo: Tropacuod aérico, el que generalmente está destinado para protección.

- Molisoles.- Los M olisoles son suelos que presentan un epipedón mélico (alto contenido en materia orgánica, suaves cuando están secos y mayor de $50 \%$ de saturación de bases), aunque no todos los suelos con este epipedón son molisoles; se excluyen los suelos con horizonte argílico, que tienen una saturación de bases menor de 35\%, etc. Ocupan áreas muy pequeñas y son poco frecuentes.

En la Región se han identificado dos (2) subgrupos: Hapludol típico y Hapludol éutrico; siendo el eutrico el que presenta mayor fertilidad natural.

3.2 Clasificación su capacidad de uso mayor

\section{- $\quad$ Tierras aptas para cultivos en limpio (A)}

Este grupo representa las tierras de mayor valor agrícola de la Región, debido a su gran capacidad productiva, abarcando una superficie aproximada de $540,000 \mathrm{Ha}$, que representa el $1.57 \%$ del total de la Región $\left(34{ }^{\prime} 456,100 \mathrm{Ha}\right.$. ), y al $11 \%$ de tierras aptas para cultivos en limpio a nivel nacional (4'902,000 Ha.). Estas tierras se encuentran fundamentalmente ocupando las áreas fisiográficas denominadas complejo de orillares, expuestos a las inundaciones de los grandes ríos de la amazonia peruana, tales como el Huallaga, U cayali, $M$ arañón y A mazonas. L a fertilidad natural de estos suelos es realmente buena, razón por Ja cual permiten la fijación de cultivos temporales o en limpio, sin mayores y sofisticados tratamientos agrícolas; por su riesgo a inundación, son consideradas como tierras de calidad agrológica baja (A 3i).

\section{- Tierras aptas para cultivos permanentes (C)}

Este grupo conjuntamente con las tierras de aptitud para cultivos en limpio, representan el potencial de tierras de la agricultura regional.

En la Región se han identificado aproximadamente $607,000 \mathrm{Ha}$, que equivale al $1.76 \%$ del total de la Región (34'456,100 Ha.) y al $22.4 \%$ de tierras aptas para cultivos permanentes a nivel nacional $\left(2^{\prime} 707,000 \mathrm{Ha}\right.$. ).

Se encuentran distribuidas en zonas denominadas lomadas, terrazas (bajas, medias y altas) y en colinas bajas, agrupando suelos de calidad agrológica de media a baja (C2 y C3), y presentando limitaciones de tipo edáfico y topográfico, como son por drenaje, suelo y erosión (C 3s, C3es y C 3 SW). 


\section{- Tierras aptas para pastos (P)}

Este grupo el tercero en extensión después de las tierras de protección y forestales, reúne aquellos suelos que presentan vocación para pastos, por ende para el desarrollo de una actividad pecuaria; abarcan una superficie de $2^{\prime} 229,000 \mathrm{Ha}$, equivalente al $6.47 \%$ del total regional $\left(34^{\prime} 456,100 \mathrm{Ha}\right.$.), y al $12.4 \%$ de tierras aptas para pastos a nivel clasificación de los pedones en si, no puede llevar a una clasificación de los cuerpos naturales del suelo.

Para poder llegar a una buena clasificación de paisajes y suelos para el mapa, se tiene que dar otras características y no sólo aquellas de los pedones; no hablar únicamente de horizontes de diagnóstico y nombres taxonómicos, sino estudiar también todos los aspectos externos o aspectos flsiográficos de los cuerpos de suelos.

Se debe admitir que los suelos tienen ciertos patrones naturales, es decir, que existe una relación estrecha entre la fisiografía yios cuerpos naturales del suelo, incluyendo los pedones individuales.

\subsection{Suelos de la Región del Amazonas según su posición fisiográfica}

Se han analizado dieciséis (16) estudios de inventario y evaluación de suelos que se realizaron en la jurisdicción de la R egión del -A mazonas. Estos estudios, generalmente desarrollados a nivel de Reconocimiento y de Semi detalle, cubren una superficie total de $8553,207 \mathrm{Ha}$., que representan el $24.81 \%$ del territorio regional. $L$ as áreas donde se concentraron estos estudios han sido: Iquitos - Nauta - Requena, Yurimaguas, Contamana, Santiago - M orona, Pastaza - Tigre, Napo - Putumayo, Caballococha y Tamshiyacu - Indiana. En el Cuadro №. 1 se presentan las diversas unidades fisiográficas identificadas en los estudios en referencia, incluyendo los suelos tipos que se encuentran en estas unidades. D el análisis de esta información se puede deducir que existe cierto nivel de correlación entre los suelos de la Región del A mazonas y las unidades fisiográficas, así se observa lo siguiente:

- En orillares e islas: predominan los Entisoles, habiéndose reconocido tropofluvent típico en áreas más elevadas (Restingas), Tropofluvent ácuico y Tropacuents en áreas de mal drenaje (Bajiales). Los suelos ubicados en la Ilanura inundable, originados por los ríos de origen andino, como el A mazonas, U cayali, M arañón y Huallaga, son los que presentan mayor fertilidad natural, en comparación con los suelos formados por sedimentos que provienen del mismo llano amazónico (Ejem: ríos Itaya y Nanay) 0 de los Andes Ecuatorianos, donde predominan rocas ácidas (Ejem: río Napo y Putumayo). 
En esta unidad fisiográfica se ubican suelos que poseen mayor vocación para cultivos en limpio, asociados a forestales y protección.

- En terrazas bajas de inundación periódica: también predominan los Entisoles, pero asociados con Inceptisoles. Los Tropofluvent y Tropacuent son los que dominan el escenario de los Entisoles, y poseen vocación para cultivos en limpio, forestales y protección. En cambio, los Inceptisoles (Distropept) son generalmente de vocación forestal.

- En terrazas bajas de inundación eventual: se observa mayor nivel dc evolución de los suelos, predominan los Inceptisoles (Tropacuept y Distropept) y poseen vocación forestal, con algunas zonas para pastos o protección.

En esta unidad también sc han identificado Histosoles (tropofibrist hídrico), con vocación forestal y algunos Entisoles con vocación agrícola, pastos y forestal.

- En terrazas medias: presentan diferente grado de disectación, también predominan los Inceptisoles, pero con mayor variabilidad a nivel de Subgrupos. LoS Distropept típico y Distropept fluvéntico, que poseen mayor vocación para cultivos permanentes y pastos; los Eutropept para cultivos en limpio los Tropacuept típico para forestal y pastos; ylos Tropacuept hístico parn protección. En esta unidad se han identificado Enti soles: Tropopsamment típico para pastos y Cuarzipsamment típico y spódico para forestales y protección. Asimismo, Ultisoles con vocación predominante para cultivos permanentes, pastos y forestales, se observa en terrazas medias, asociadas a Alfisoles (Paleudalf con vocación para cultivos en limpio y cultivos permanentes. En la zona de Contamana se ha identificado pequeñas áreas con Molisol (Hapludol éutrico) con vocación para cultivos en limpio y cultivos permanentes.

- En terrazas altas: se encuentra una asociación predominante de UItisoles e Inceptisoles. Dentro del primero se observa Tropudult y Paleudult, que son aptos para cultivos permanentes, pastos y forestales. En Inceptisolns se ha identificado Distropept, que tiene una vocación para cultivos permanentes y Tropacuept con vocación forestal. En esta unidad fisiográfica también existe pequeñas áreas con Entisoles arenosos (Tropopsamment y Quazipsamment) y Podsoles (Tropacuod aérico), que son para protección.

- En lomadas: se han identificado con mayor incidencia Inceptisoles, destacando Distropept y Eutropept, con vocación para cultivos permanentes, pastos y forestales.

- En vallecitos: intercolinosos: se presentan Entisoles (Troportent y Tropofluvent) con aptitud para cultivos en limpio, forestales y protección. 
También se reporta Inceptisoles: Distropept típico para pastos y Tropacuept típico para forestal.

- En colinas bajas y medias: predominan los Inceptisoles asociados con Ultisoles. En el primero se reportan Distropept y Eutropept con vocación para pastos, cultivos permanentes y forestales. En los Ultisoles, que poseen más vocación forestal, se registra Tropudult y Paleudult. En esta unidad fisiográfica también se encuentran Alfisoles (Tropudalf), con vocación forestal yT ropopsamment típico, con aptitud para cultivos permanentes.

A Igunos M olisoles se reportan en la zona de Contamana.

- En cerros bajos y montañas: se reportan Entisoles (Troportent lítico) e Inceptisoles (Distropept lítico y Eutropept Iftico); se considera que estas tierras, por estar situadas en fuertes pendientes, son de protección. 


\section{CUADRO № 1}

\section{SUELOS DE LA REGION DEL AMAZONAS SEGUN UNIDADES FISIOGRAFICAS}

Unidades Fisiográficas Clasificación Taxonómica $\begin{gathered}\text { Capacidad de Uso } \\ \text { Mayor }\end{gathered}$

\begin{tabular}{|c|c|c|c|c|}
\hline & Orden & Gran Grupo & Sub Grupo & \\
\hline \multirow{3}{*}{$\begin{array}{l}\text { Orillares, Islas y } \\
\text { Emplayamientos }\end{array}$} & \multirow{3}{*}{ ENTISOL } & \multirow[t]{2}{*}{ Tropofluvent } & - típico & $\begin{array}{l}\text { C. Limpio (A2si, A3iw) } \\
\text { C. Permanent. (C3iw) } \\
\text { Forestales (F2iw) } \\
\text { Protección (Xiw) }\end{array}$ \\
\hline & & & \multicolumn{2}{|l|}{ - ácuico } \\
\hline & & Tropacuents & - típico & $\begin{array}{l}\text { Cult. Limpio (A3wi) } \\
\text { Protección }(X)\end{array}$ \\
\hline \multirow{6}{*}{$\begin{array}{l}\text { Terrazas Bajas } \\
\text { de } \\
\text { Inundación Periód }\end{array}$} & \multirow{3}{*}{$\begin{array}{l}\text { ENTISOL } \\
\text { dica }\end{array}$} & Tropofluvents & - típico & $\begin{array}{l}\text { C. Limpio (A2si, A3wi) } \\
\text { Pastos (P3s) } \\
\text { Forestal (F2iw) }\end{array}$ \\
\hline & & & - ácuico & $\begin{array}{l}\text { C. Limpio (A3si) } \\
\text { Protección (Xsi) }\end{array}$ \\
\hline & & Tropacuents & .............. & $\begin{array}{l}\text { Forestal (F3se) } \\
\text { Protección }(X)\end{array}$ \\
\hline & \multirow[t]{3}{*}{ INCEPTISOL } & Distropepts & - típico & Forestal (F2siw) \\
\hline & & Tropacuent & ................ & Forestal (F3se) \\
\hline & & Tropofluvents & ............... & C. Limpio (As, A2si) \\
\hline
\end{tabular}


Terrazas Bajas

ENTISOL Troportens

- típico

C. Limpio (A3)

Tropofluvent -típico Pastos (P3s)

de Inundación

- molico

C. Limpio (A3sw)

-aérico Forestal (F3isw)

Tropacuepts

-típico Forestal (F3swi)

Eventual

\begin{tabular}{clll}
\hline INCEPTISOL & Distropepts & -típico & Pastos (P2s) \\
\hline HISTOSOL & Tropofibrist & - hídrico & Forestal (F3wis) \\
\hline
\end{tabular}

$+2$

Terrazas

\begin{tabular}{|c|c|c|c|}
\hline \multirow{4}{*}{ ENTISOL } & \multicolumn{2}{|c|}{ Tropopsamment - típico } & \multirow{4}{*}{$\begin{array}{l}\text { Pastos (P3s) } \\
\text { Forestal (Fs) } \\
\text { Protección }(X) \\
\text { Protección }(X)\end{array}$} \\
\hline & & = típico & \\
\hline & \multicolumn{2}{|c|}{ Cuarzipsamment } & \\
\hline & & - spódico & \\
\hline \multirow{10}{*}{ INCEPTISOL } & \multirow{4}{*}{ Tropacuept } & - típico & Pastos (P2sw, P2s) \\
\hline & & & Forestal (F2sw, F3si) \\
\hline & & & Protección $(X)$ \\
\hline & & - hístico & Protección (X) \\
\hline & \multirow{6}{*}{ Distropept } & - típico & C. Limpio (As) \\
\hline & & & C. Permat. (C2e, C3si \\
\hline & & & Pastos (P2s, P3sw) \\
\hline & & -fluvéntico & C. Limpio (A2s, A3ss \\
\hline & & & C. Permat. (C3se) \\
\hline & & & Pastos (P3se) \\
\hline
\end{tabular}

Medias

\begin{tabular}{llll} 
& Eutropept & $\ldots \ldots \ldots \ldots . .$. & C. Limpio (A2s) \\
\hline \multirow{2}{*}{ MOLISOL } & Hapludol & - eutrico & C. Limpio (A3se) \\
& & & C. Permat. (C2) \\
\hline \multirow{2}{*}{ ALFISOL } & Paleudalf & $\ldots \ldots \ldots \ldots$ & $\begin{array}{l}\text { C. Limpio (A) } \\
\text { Pastos (P) }\end{array}$ \\
\hline & & & $\begin{array}{l}\text { C. Limpio (Asi) } \\
\text { C. Permat. (Ces) } \\
\text { Pastos (P) }\end{array}$ \\
& & Paleudult & - típico
\end{tabular}




\begin{tabular}{|c|c|c|c|c|}
\hline & \multirow{3}{*}{ ULTISOL } & \\
\hline & & Plintacult & .............. & Forestal (F) \\
\hline & & Plintudult & .............. & Pastos (P) \\
\hline \multirow[b]{5}{*}{ Terrazas } & \multirow{3}{*}{ ENTISOL } & \multirow{2}{*}{\multicolumn{2}{|c|}{ Quarzipsamment - spódico }} & Forestal $(\mathrm{F})$ \\
\hline & & & & Proteccion $(X)$ \\
\hline & & \multicolumn{2}{|c|}{ Tropopsamments - típico } & Forestal (Fs) \\
\hline & & Tropacuepts & $\begin{array}{l}\text { - típico } \\
\text { - aérico }\end{array}$ & $\begin{array}{l}\text { Forestal (F3sw) } \\
\text { Forestal (F3sw) }\end{array}$ \\
\hline & & Distropepts & $\begin{array}{l}\text { - típico } \\
\text { - fluvéntico }\end{array}$ & $\begin{array}{l}\text { C. Permanent. (C3s) } \\
\text { Pastos (P3s) } \\
\text { C. Permanent. (C3s) }\end{array}$ \\
\hline \multirow[t]{4}{*}{ Altas } & \multirow{3}{*}{ ULTISOL } & Tropudult & - típico & $\begin{array}{l}\text { C. Limpio (As) } \\
\text { C. Permat. (C2es) } \\
\text { Pastos (P2s) }\end{array}$ \\
\hline & & Tropudult & - ortóxico & $\begin{array}{l}\text { C. Permat. (C3s) } \\
\text { Pastos (P2s) } \\
\text { Forestal (Fs) }\end{array}$ \\
\hline & & Paleudult & - típico & $\begin{array}{l}\text { C. Limpio (As) } \\
\text { C. Permat. (Ces) } \\
\text { Forestal (Fes) }\end{array}$ \\
\hline & PODSOL & Tropacuod & - aérico & Protección \\
\hline \multirow{3}{*}{ Lomadas } & ENTISOL & Troportent & - típico & Forestal (F2e, F3e) \\
\hline & & Eutropept & $\begin{array}{l}\text { - típico } \\
\text { - fluvéntico }\end{array}$ & $\begin{array}{l}\text { Forestal }(\mathrm{F} 2 \mathrm{e}) \\
\text { C. Permat. (C2) }\end{array}$ \\
\hline & INCEPTISOL & Distropept & - fluvéntico & $\begin{array}{l}\text { Pastos (P3se) } \\
\text { Forestal (F1se) } \\
\text { C. Limpio (A3s) } \\
\text { C. Permanent. (C3es) } \\
\text { Pastos (P3es) } \\
\text { Forestal (F2es) }\end{array}$ \\
\hline
\end{tabular}




\begin{tabular}{|c|c|c|c|c|}
\hline & ULTISOL & Paleudult & - típico & $\begin{array}{l}\text { C. Limpio (Asl) } \\
\text { C. Permat. (Cesl, C2) } \\
\text { Pastos (P2s) }\end{array}$ \\
\hline \multirow{4}{*}{$\begin{array}{l}\text { Vallecitos } \\
\text { Intercolinosos }\end{array}$} & \multirow[b]{2}{*}{ ENTISOL } & Tropofluvent & .............. & Proteccion (Xs) \\
\hline & & Troportent & - típico & $\begin{array}{l}\text { C. Limpio (A3) } \\
\text { Forestal (F2es) }\end{array}$ \\
\hline & \multirow{2}{*}{ INCEPTISOL } & Tropacuepts & - típico & Forestal (F2isw) \\
\hline & & Distropept & - típico & Pastos (Psw) \\
\hline \multirow[b]{3}{*}{ Colinas } & \multirow[t]{2}{*}{ ENTISOL } & Tropopsamme: & t - típico & C. Permanent. (C) \\
\hline & & Eutropept & - típico & Forestal (F2e, F3e) \\
\hline & INCEPTISOL & Distropept & - típico & $\begin{array}{l}\text { C. Limpio (A3s) } \\
\text { C. Permenent. (C3es) } \\
\text { Pastos (P3s) } \\
\text { Forestal (F2es, F3es) }\end{array}$ \\
\hline \multirow[t]{4}{*}{$\begin{array}{l}\text { Bajas } \\
\mathrm{Y} \\
\text { Medias }\end{array}$} & MOLISOL & Hapludol & $\begin{array}{l}\text { - típico } \\
\text { - eutrico }\end{array}$ & $\begin{array}{l}\text { Pastos (P2es) } \\
\text { Forestal (F2e, F3e) } \\
\text { Pastos (P2es) } \\
\text { Forestal (F2e, F3e) }\end{array}$ \\
\hline & ALFISOL & Tropudalf & $\ldots \ldots \ldots \ldots$ & Forestal (F2es) \\
\hline & \multirow{2}{*}{ ULTISOL } & Tropodult & ............. & Forestal (F2es) \\
\hline & & Paleudult & ............... & Forestal (F) \\
\hline \multirow{3}{*}{$\begin{array}{l}\text { Cerros Bajos } \\
\text { Y } \\
\text { Montañas }\end{array}$} & ENTISOL & Troportent & - lítico & Protección $(X)$ \\
\hline & \multirow{2}{*}{ INCEPTISOL } & Eutropept & - lítico & Protección (Xes) \\
\hline & & Distropept & - lítico & Protección (Xes) \\
\hline
\end{tabular}

